\title{
Efficient Approximation of the Mahalanobis Distance for Tracking with the Kalman Filter
}

\author{
Raquel R. Pinho \\ FEUP - Faculdade de Engenharia da Universidade do Porto, Portugal \\ INEGI - Instituto de Engenharia Mecânica e Gestão Industrial
}

João Manuel R. S. Tavares

FEUP (DEMEGI - Dep. de Engenharia Mecânica e de Gestão Industrial), INEGI

Miguel V. Correia

FEUP (DEEC - Dep. de Engenharia Electónica e de Computadores)

INEB - Instituto de Engenharia Biomédica

\begin{abstract}
We address the problem of tracking efficiently feature points along image sequences. To estimate the undergoing movement we use an approach based on Kalman filtering which performs the prediction and correction of the features movement in every image frame. In this paper measured data is incorporated by optimizing the global correspondence set based on efficient approximations of the Mahalanobis distances (MD). We analyze the difference between using the MD and its efficient approximation in the tracking results, and also examine the related computational costs. Experimental results which validate our approach are presented.
\end{abstract}

\section{INTRODUCTION}

Feature tracking is a complex problem whose automatic detection and execution evolved considerably in the past decade. Applications of movement tracking are numerous: surveillance, object deformation analysis, traffic monitorization, etc (see for example (Wren, Azarbayejani, Darell, and Pentland 1997; Cucchiara, Grana, Piccardi, and Prati 2000)). In the case of human movement analysis it can be used in medical diagnosis, physical therapy or sports, for example to study gait disorders related to knee or hip injury or pain, to control cycles of motion in rehabilitation or training processes.

Although the computational performance has improved significantly, the tracking systems which are able to capture and analyze the undergoing movement, for instance to track multiple features simultaneously or to obtain on-line results, often use some kind of simplification to speedup the process. So, a compromise must be achieved between the accuracy of the motion estimation and the related computational cost.

The Pfinder (Wren, Azarbayejani, Darell, and Pentland 1997) is a real-time system for tracking people and interpreting their behavior. It does not require accurate initialization and segments the person from the background in real-time with a standard computer workstation, but it expects only one person to be in the image scene, and the scene is supposed to be significantly less dynamic than the tracked person.

In (Jorge, Abrantes, and Marques 2004) Bayesian networks are used to perform tracking even in occlusions, group formation and splitting. The on-line object tracking is performed by gradually discarding the influence of past information on the current decisions avoiding a combinatorial explosion and keeping the network complexity within reasonable bounds.

On the other hand, many current works use a probabilistic representation of the uncertainty and stochastic filters to fuse and validate sensor information, and to estimate parameters describing the environment. In (Lin and Bar-Shalom 2004) the data association problem is combined with filtering to track ground targets using ground moving target indicator (GMTI) reports obtained from an airborne sensor.

The matching between the estimated features and the observations detected after a sensing operation is determined using data association techniques. Data association algorithms may include a hypothesisvalidation step, which may be based on the Maha- 
lanobis distance (MD) and its validation through the $\chi^{2}$-distribution.

\subsection{Related Work}

The use of the MD for associating data in tracking systems is usual as it gives good performance, but few approaches have tried to speedup this procedure. In (O'Malley, Nechyba, and Arroyo 2002) is presented a method for tracking and identifying moving persons from video images taken by a fixed field-of-view camera, and the MD is used to distinguish pixels either from foreground or background. To speed the calculation only the diagonals of the covariance matrix are used in the MD calculation. This corresponds to the same simplification we use, but applied in different tracking approaches.

On the other hand, the MD and its simplifications extensively are used for pattern recognition. As the computation time of the MD will reach $O\left(n^{2}\right)$ for $n$-dimensional feature vectors, to reduce the computational cost several approximations of the MD have been proposed as, for example, the quasiMahalanobis distance (Kurita, Tsuruoka, Yokoi, and Miyake 1983), the modified Mahalanobis distance (Kato, Abe, and Nemoto 1996), and the modified quadratic discriminant function (Kimura and Shridhar 1991). All these approaches were proposed to improve recognition accuracy but not to approximate the quadratic discriminant function.

In (Sun, Omachi, Kato, Aso, Kono, and Takagi 2000) an approximation of the MD is used in pattern recognition for example in Chinese and Japanese character recognition. Based on two kinds of approximations of the MD, the proposed algorithm consists of two stages which are feature vector division and dimensional reduction. The first stage of feature division is based on the characteristic of covariance matrix. The second stage is done by regarding the values of small eigenvalues as a constant. When compared to the well-known dimensional reduction method, the Karhunen-Loéve (K-L) expansion, experimental results showed that the proposed algorithm not only reduced the computational cost but also improved the recognition accuracy. But this approach requires the computation of the involved eigenvalues.

\subsection{Our Approach}

In our previous work we used the MD to evaluate the quality of correspondences, and the actual set of matching between predictions and measurements is obtained by optimizing the sum of all the involved MD (Pinho, Tavares, and Correia 2005). By doing so, the best global correspondence set is guaranteed. To track the captured movement we use a well-known statistical model: the Kalman filter (KF). The drawbacks of this stochastic process are due to its relatively high restrictive assumptions (Welch and Bishop 1995). But we combined the KF with optimization techniques for data association in order to increment the filters robustness to occlusion and nonlinear movement. The correspondence between each features prediction and new measurement data is set upon the MD minimization. The MD ensures that the correspondence is done according to each features previously known behavior. Its approximation to the $\chi^{2}$-distribution allows the choice of a significance level, from which features will be considered as unmatched. Therefore, even if the KF restrictions are not satisfied (a often situation in many tracking applications) the results obtained may be corrected with optimization techniques and the MD.

In this paper we propose an efficient approximation of the MD between features, in order to sort them and match corresponding features along image sequences for tracking. Experimental results which validate our approach are presented.

\subsection{Paper Overview}

This paper is organized as follows. In the next section a brief introduction is made to the MD. In section 3, we describe the used efficient approximation to the $\mathrm{MD}$ and analyze the undergoing error. In section 4 we overview the tracking system which uses the efficient approximation of the MD for features correspondence along frames. In section 5 some experimental results are shown on synthetic and real image sequences. In the last section some conclusions will be held.

\section{THE MAHALANOBIS DISTANCE}

The MD, also known as statistical distance, is a distance that for each of its components (the variables) takes the variability of that variable into account when determining its distance from the center. So, components with high variability receive less weight than components with low variability. This is done obtained by rescaling the components. That is, for two points $X_{i}=\left(x_{1 i}, x_{2 i}, \ldots, x_{n i}\right)$ and $Y_{j}=$ $\left(y_{1 j}, y_{2 j}, \ldots, y_{n j}\right)$ the MD is given by

$$
d_{M}=\sqrt{\left(X_{i}-Y_{j}\right)^{T} C^{-1}\left(X_{i}-Y_{j}\right)}
$$

where $C_{(n \times n)}$ is a non-singular covariance matrix (therefore symmetric positive definite). Points with the same distance of $X_{i}$ satisfy the equation of an ellipsoid centered about $X_{i}$, and those points of the ellipsoid defined by $C$ have unitary MD.

The MD is a standard manner of associating data for tracking features in image sequences. However, this step is one of the most time-consuming operations of the matching process. After a sensing operation, $M$ feature location estimates, and $N$ measurements, are available. The problem is how to associate 
each measurement, $X_{i}(i=1, \ldots, N)$, with a feature estimate, $Y_{j}(j=1, \ldots, M)$.

This matching procedure is time-consuming because it involves a matrix inversion, and the computation of matrix $C$ and vector $v=X_{i}-Y_{j}$ which is subject to linearizations. To save computational cost some data-association techniques perform a validation test for each pairing hypothesis, in order to work with only a reduced set of hypotheses. The process of the validation is performed using a statistical test based on the MD and its approximation by the $\chi^{2}$ distribution:

$$
v^{T} C^{-1} v \leq \chi^{2}
$$

where $v$ is the vector between a predicted feature state and an acquired measurement. This test should theoretically be computed for $M \mathrm{x} N$ hypotheses.

\section{AN EFFICIENT APPROXIMATION OF THE MAHALANOBIS DISTANCE}

In our tracking system the captured measurements are composed by their position coordinates in the image plane, and each of the tracked features has its own KF. This means that the $C$ and $v$ are given by:

$$
C=\left[\begin{array}{ll}
c_{11} & c_{12} \\
c_{12} & c_{22}
\end{array}\right]
$$

and,

$$
V=\left[\begin{array}{ll}
v_{1} & v_{2}
\end{array}\right]^{T} .
$$

So the MD in this case is given by:

$$
d_{M}=\frac{c_{22} v_{1}^{2}-2 c_{12} v_{1} v_{2}+c_{11} v_{2}^{2}}{c_{11} c_{22}-c_{12}^{2}} .
$$

Rearranging the terms in the equation above we can obtain

$$
\begin{aligned}
d_{M} & =\frac{v_{1}^{2}}{c_{11}}+\frac{v_{2}^{2}}{c_{22}}-\frac{2 c_{12} v_{1} v_{2}}{c_{11} c_{22}}+ \\
& +\frac{c_{12} c_{22} v_{1}^{2}+c_{12} c_{11} v_{2}^{2}-2 c_{12}^{3} v_{1} v_{2}}{c_{11} c_{22}\left(c_{11} c_{22}-c_{12}^{2}\right)} .
\end{aligned}
$$

So, the efficient approximation we propose to the MD consists on:

$$
d_{M} \approx \hat{d_{M}}=\frac{v_{1}^{2}}{c_{11}}+\frac{v_{2}^{2}}{c_{22}},
$$

which is thereby affected of an error, $\Delta \hat{d_{M}}$, of

$\Delta \hat{d_{M}}=-\frac{2 c_{12} v_{1} v_{2}}{c_{11} c_{22}}+\frac{c_{12} c_{22} v_{1}^{2}+c_{12} c_{11} v_{2}^{2}-2 c_{12}^{3} v_{1} v_{2}}{c_{11} c_{22}\left(c_{11} c_{22}-c_{12}^{2}\right)}$

Although better approximations can be used for the MD their computational cost can be questioned, on the other hand, the approximation that we propose is quite efficient as it only involves 5 arithmetic operations for each pair of features; instead of the 18 operations involved in equation (5) with the usual calculation of the MD.

\section{TRACKING WITH THE KALMAN FILTER AND ASSOCIATING DATA WITH THE MA- HALANOBIS DISTANCE}

The KF is an optimal recursive Bayesian stochastic method. It provides optimal estimates that minimize the mean of squared error of the modeled process. In a Bayesian stochastic viewpoint, the filter propagates conditional probability density of the system state conditioned on the knowledge of the actual data acquired by the measuring devices.

The equations for the KF fall into two steps: time update (or prediction) and measurement update (or correction). The time update equations are responsible for projecting forward (in time) the current state and error covariance estimates to obtain the a priori estimates for the next time step. The measurement update equations deal with the feedback, that is new measurements are incorporated into the $a$ priori estimates to obtain improved a posteriori values (Welch and Bishop 1995).

The prediction step is based on the ChapmanKolmogorov equation for a first order Markov process:

$$
x_{t}^{-}=\Phi x_{t-1}^{+}
$$

where $\Phi$ relates the system state $x_{t-1}^{+}$at the previous time step $t-1$ to the state $x_{t}^{-}$at the current step $t$. The superscripts ${ }^{+}$and ${ }^{-}$indicate if measurement data have been or not incorporated, respectively. The related uncertainty is given by:

$$
P_{t}^{-}=\Phi P_{t-1}^{+} \Phi^{T}+Q
$$

where $P$ is the prediction covariance matrix and $Q$ models the process noise.

The correction equations that update the predicted estimates upon the incorporation of new $u_{t}$ measurements are given by:

$$
\begin{gathered}
K_{t}=P_{t}^{-} H^{T}\left[H P_{t}^{-} H^{T}+R_{t}\right]^{-1} \\
x_{t}^{+}=x_{t}^{-}+K_{t}\left[u_{t}-H x_{t}^{-}\right] \\
P_{t}^{+}=\left[I-K_{t} H\right] P_{t}^{-}
\end{gathered}
$$

where $K$ is chosen to be the gain that minimizes the a posteriori error covariance equation, $H$ processes the coordinates transformation between the predicted and the measurement spaces, $R$ is the measurement noise involved, and $I$ is the identity matrix (Welch and Bishop 1995). 
One of the drawbacks of the KF is the restrictive assumption of Gaussian posterior density functions at every time step, as many tracking problems involve non-linear movement. To minimize such ambiguities we evaluate all the $M \times N$ possible correspondences by estimating the MD, and optimize the global matching set. The efficient MD can sort correspondences according to their MD, and so the computational burden associated to the MD calculation in the matching process is overcome with a small error involved.

\section{EXPERIMENTAL RESULTS}

In each frame of the presented examples the predicted position is represented with $\mathrm{a}+$, with uncertainty area circumscribed in a solid ellipse, each measurement is the center of the detected contour, and the corrected position is represented by a $\mathrm{x}$. The association between each prediction/measurement is represented with a solid line.

For the first example, Figure 1, consider a synthetic sequence of 9 frames. In the beginning of the sequence only two blobs are visible. The circular blob will disappear definitively but the tracking approach keeps on trying to track it during the subsequent frames, although with gradually higher uncertainty (in frame (e) the uncertainty region surpasses the image border). In the second frame a triangular blob appears, and in the third frame the square blob disappears instantly. In the fourth frame the captured blobs overlap, and with the used image processing techniques only one measurement is captured and associated to a blob, but both features continue to be correctly tracked. From the seventh frame onwards 25 blobs are tracked. The results presented in Figure 1 were obtained by data association with the efficient MD but there exists no visual difference to those obtained with the usual MD calculation, but the computational cost associated to the efficient MD is obviously less, Figure 2. Indeed, as little features are tracked the computational load of the MD is not significant, but as the number of tracked features increases the advantages of the efficient MD are more notable.

In Tables 1 and 2 are represented the results obtained with the efficient approximation of the MD and those obtained with the usual MD, respectively, to associate the tracked features and the measurements in frame (d) of Figure 1. It can be noticed that the efficient approximation of the MD can be larger or smaller than the usual value, but differences are generally small, Figure 3.

Note that in frame (d) of Figure 1 three features are being tracked while only two measurements are captured, so to apply the optimization algorithm a fictitious variable is included, and the cost of correspondence with it is null (Pinho, Tavares, and Correia 2005).
Minimizing the overall correspondence costs evaluated in our tracking approach with the MD or its efficient approximation the same results are obtained: the third tracked feature, $X e_{3}$ is associated to the first measurement captured; $X m_{1}$; the second tracked feature and measurement are matched, $X e_{2}$ and $X m_{2}$ respectively; and the first tracked feature $X e_{1}$ is associated to the fictitious variable $X m_{3}$, which means that it is considered unmatched (Tables 1 and 2).

What is more advantageous to our tracking application is that the efficient approximation of the MD can efficient and adequately sort out the features to build the correct correspondences along the image sequences.

Table 1: Associating tracked features and measurements in Figure 1 frame (d): Values of the efficient approximation of the MD.

\begin{tabular}{|c|l|l|l|}
\hline & \multicolumn{3}{|c|}{ Measurements } \\
\hline Tracked Features & $X m_{1}$ & $X m_{2}$ & $X m_{3}$ \\
\hline$X e_{1}$ & 5.635 & 7.248 & $\mathbf{0 . 0}$ \\
$X e_{2}$ & 3.910 & $\mathbf{1 . 6 7 8}$ & 0.0 \\
$X e_{3}$ & $\mathbf{0 . 4 0 6}$ & 2.570 & 0.0 \\
\hline
\end{tabular}

Table 2: Associating tracked features and measurements in Figure 1 frame (d): Values of the MD.

\begin{tabular}{|c|c|c|l|}
\hline & \multicolumn{3}{|c|}{ Measurements } \\
\hline Tracked Features & $X m_{1}$ & $X m_{2}$ & $X m_{3}$ \\
\hline$X e_{1}$ & 5.930 & 7.816 & $\mathbf{0 . 0}$ \\
$X e_{2}$ & 3.652 & $\mathbf{1 . 6 5 7}$ & 0.0 \\
$X e_{3}$ & $\mathbf{0 . 3 8 2}$ & 2.416 & 0.0 \\
\hline
\end{tabular}

For the next example consider a sequence of real images presented in (Brown, Senior, Tian, Connell, Hampapur, Shu, Merkl, and Lu 2005) for the evaluation of performance of surveillance systems. In this example a person walks into the room and stops at the center, meanwhile two other persons enter, walk around the room and exit. Then, the remaining person walks out the room.

One usual difficulty related to tracking a person with most low level image processing techniques is the detection of 2 regions of movement, one of them corresponding to the peoples head and upper body, and the other to their legs and feet. In the images presented we did not try to remove those and other instantaneous noisy features in order to test the proposed data association algorithm. We tracked the moving features using both the MD as well as its efficient approximation and generally the same results are obtained, Figure 4. But in some rare cases the data association may differ as in Figure 5, but in the next frames the tracking process is correctly recovered. 


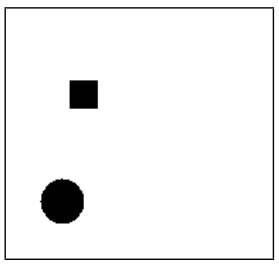

(a)

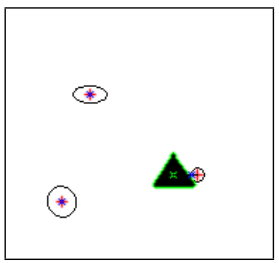

(c)

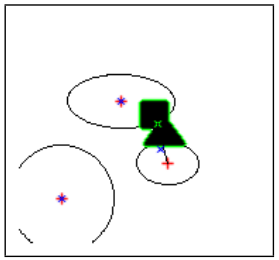

(e)

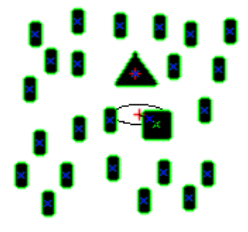

(g)

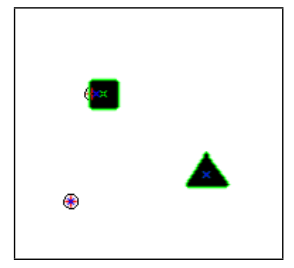

(b)

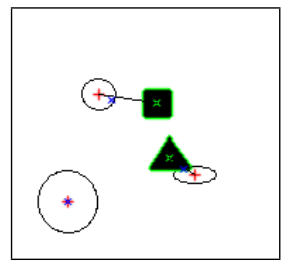

(d)

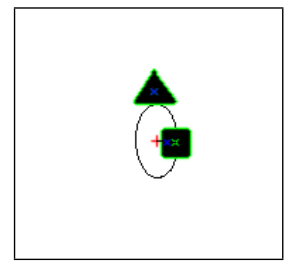

(f)

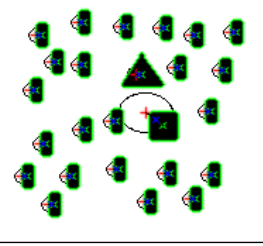

(h)

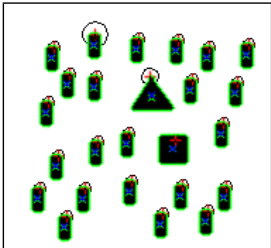

(i)

Figure 1: Tracking blobs in a 9 frame image sequence: (a) - original $1^{\text {st }}$ frame; (b) to (i) - KF results: search area defined by solid ellipses, the predicted position for each marker is given by + , and the corrected position is represented with $\mathrm{x}$.

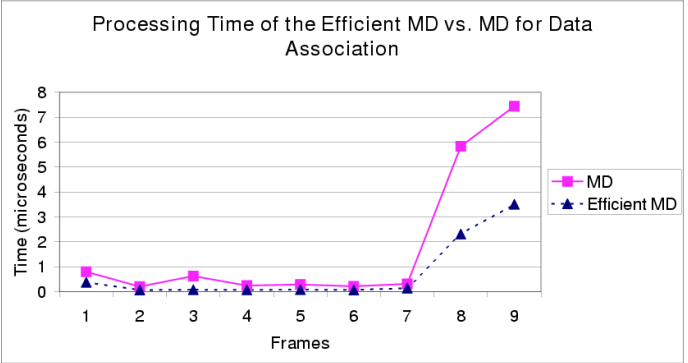

Figure 2: Data association time while using the efficient MD or the usual MD on a Mobile AMD Atlhon $(\mathrm{tm}) 4$ at $1.20 \mathrm{GHz}$ with $256 \mathrm{MB}$ RAM.

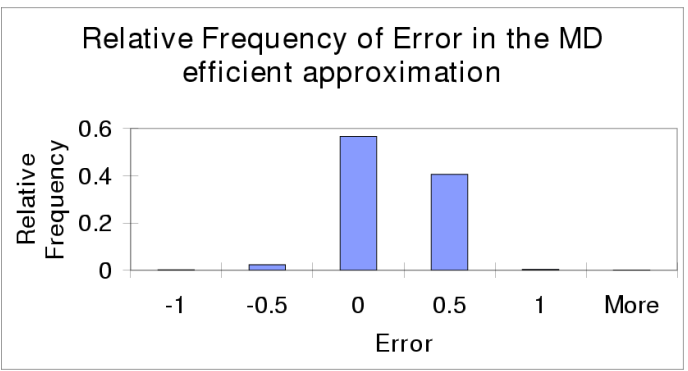

Figure 3: Relative Frequency of errors in the efficient approximation of MD.

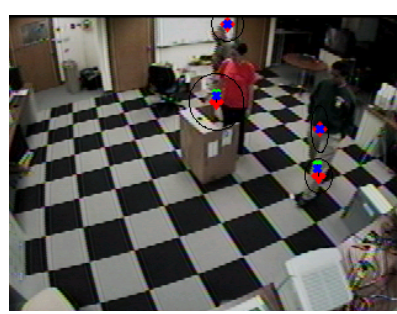

(a) frame 8

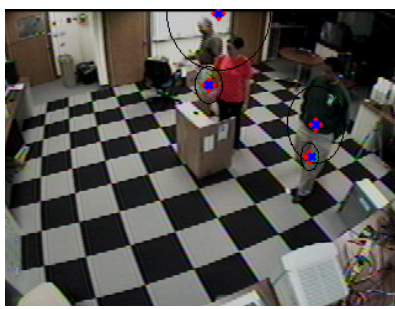

(c) frame 10

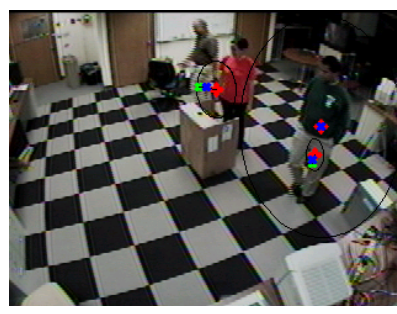

(e) frame 12

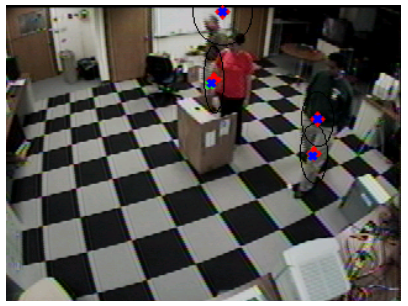

(b) frame 9

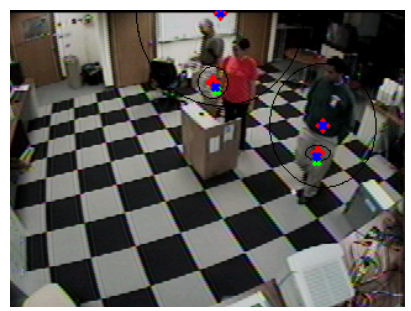

(d) frame 11

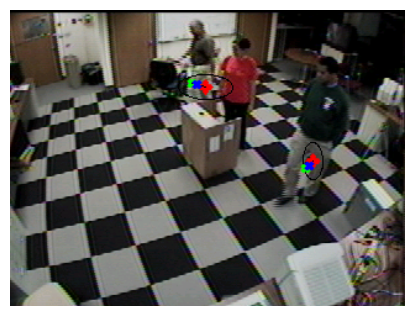

(f) frame 13
Figure 4: Tracking people in a surveillance system: results obtained with data association using the efficient approximation of the MD. 


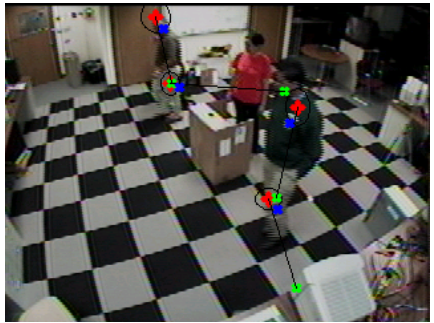

(a) frame 21

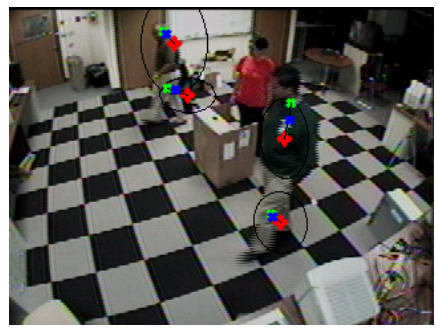

(c) frame 22

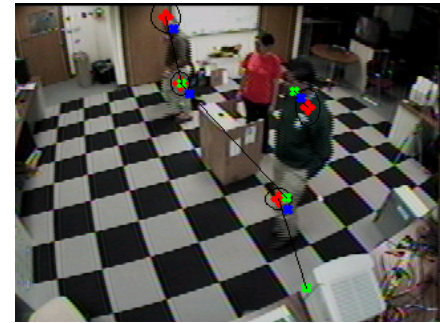

(b) frame 21

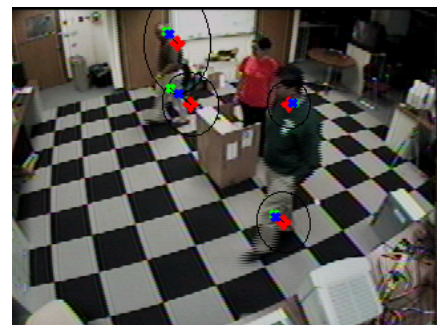

(d) frame 22
Figure 5: Results obtained with data association using the efficient approximation of the MD (left column) and the MD (right column).

\section{CONCLUSIONS}

To track features in image sequences we use a $\mathrm{KF}$ which performs the prediction and correction of the features movement in every image frame. With our approach the correspondence between each prediction made by the KF and its measurement in each frame are established by optimizing the global matching set based on the MD. To reduce the computational cost associated to the calculation of the MD we propose in this paper a simplification of the MD with an efficient approximation in order to reduce the computational cost of associating data in the KF for tracking. We have exemplified that this approach is especially advantageous when a large number of features are tracked, and have shown that generally the resulting correspondences are very similar to those obtained with the usual MD.

\section{ACKNOWLEDGMENTS}

This work was partially done in the scope of the project Segmentation, Tracking and Motion Analysis of Deformable (2D/3D) Objects using Physical Principles, reference POSC/EEA-SRI/55386/2004, financially supported by FCT - Fundação para a Ciência e a Tecnologia in Portugal.

The first author would like to thank the support of the PhD grant SFRH / BD / 12834 / 2003 of FCT.

\section{REFERENCES}

Brown, L., A. Senior, Y. Tian, J. Connell, A. Hampapur, C. Shu, H. Merkl, and M. Lu (2005). Performance evaluation of surveillance systems under varying conditions. In IEEE International Workshop on Performance Evaluation of Tracking and Surveillance, Colorado, USA.

Cucchiara, R., C. Grana, M. Piccardi, and A. Prati (2000). Statistic and knowledge-based moving object detection in traffic scenes. In IEEE Conference on Intelligent Transportation Systems, Dearborn, USA.

Jorge, P., A. Abrantes, and J. Marques (2004). On-line tracking groups of pedestrians with bayesian networks. In Workshop PETS, Prague, Czech Republic.

Kato, N., M. Abe, and Y. Nemoto (1996). A handwritten character recognition system using modified mahalanobis distance. Transactions Institute of Electronics, Information and Communication Engineers (IEICE) J79-D(1), 45-52.

Kimura, F. and M. Shridhar (1991). Handwritten numerical recognition based on multiple algorithms. Pattern Recognition 24, 969-983.

Kurita, M., S. Tsuruoka, S. Yokoi, and Y. Miyake (1983). Handprinted "kanji" and "hiragana" character recognition using weighting direction index histograms and quasi-mahalanobis distance. Technical report, IEICE Technical Report PRL82-79.

Lin, L. and Y. Bar-Shalom (2004). New assignment-based data association for tracking move-stop-move targets. IEEE Transactions on Aerospace and Electronic Systems 40(2), 714-725.

O’Malley, P., M. Nechyba, and A. Arroyo (2002). Human activity tracking for wide-area surveillance. In 2002 Florida Conference on Recent Advances in Robotics, Miami, USA.

Pinho, R., J. Tavares, and M. Correia (2005). Human movement tracking and analysis with kalman filtering and global optimization techniques. In II International Conference On Computational Bioengineering, Lisbon, Portugal.

Sun, F., S. Omachi, N. Kato, H. Aso, S. Kono, and T. Takagi (2000). Two-stage computational cost reduction algorithm based on mahalanobis distance approximations. International Journal of Pattern Recognition 2, 2969-2973.

Welch, G. and G. Bishop (1995). An Introduction to Kalman Filter. Technical report, University of North Carolina at Chapel Hill.

Wren, C., A. Azarbayejani, T. Darell, and A. Pentland (1997). Pfinder: Real-time tracking of the human body. IEEE Transactions on Pattern Analysis and Machine Intelligence 19(7), 780785. 